\title{
Aberrant methylation of secreted protein, acidic and rich in cysteine in human laryngeal and hypopharyngeal carcinoma
}

\author{
QIAN HE $^{1 *}$, JIAZHANG WEI ${ }^{2 *}$, JINYAN ZHANG $^{3}$, HENG JIANG $^{1}$, SHUMIN WANG $^{1}$, \\ XIAOYING ZHOU $^{1}$, ZHE ZHANG ${ }^{1}$, GUANGWU HUANG ${ }^{1}$, HIROSHI WATANABE ${ }^{2}$ and JIPING SU ${ }^{1}$ \\ ${ }^{1}$ Department of Otolaryngology-Head and Neck Surgery, First Affiliated Hospital of Guangxi Medical University, \\ Nanning 530021, P.R. China; ${ }^{2}$ Department of Clinical Pharmacology and Therapeutics and \\ ${ }^{3}$ Photon Medical Research Center, Hamamatsu University School of Medicine, Hamamatsu 431-3192, Japan
}

Received December 12, 2010; Accepted April 12, 2011

DOI: $10.3892 / 01.2011 .297$

\begin{abstract}
Secreted protein, acidic and rich in cysteine (SPARC) has been found to be involved in various stages of tumor progression such as migration, invasion, extracellular matrix deposition and angiogenesis. To obtain an insight into the role of SPARC in the progression of laryngeal and hypopharyngeal carcinoma, we investigated SPARC transcription levels and promoter methylation in carcinoma cell lines and primary tumors. Reverse transcription-PCR showed that SPARC was silenced in laryngeal and hypopharyngeal carcinoma cell lines, in which aberrant promoter methylation was detected. Hypermethylation of SPARC was detected in $56.1 \%(23 / 41)$ of laryngeal carcinoma and $70.0 \%(7 / 10)$ of hypopharyngeal carcinoma biopsies, but only in $11.1 \%$ (1/9) of normal epithelial specimens by a methylation-specific PCR assay. Bisulphite genomic sequencing indicated that $\mathrm{CpG}$ sites in the SPARC promoter were heavily methylated in cell lines and primary tumors. Moreover, pharmacological demethylation treatment rescued SPARC expression with 5-aza-2'-deoxycytidine (5-aza-dC) in the laryngeal carcinoma cell lines. SPARC promoter hypermethylation was significantly correlated with lymph node metastasis $(\mathrm{p}<0.01)$. Our findings suggest that hypermethylation of SPARC is a frequent and tumor-specific event in laryngeal and hypopharyngeal carcinomas and may serve as a biomolecular marker for diagnosis and prognosis.
\end{abstract}

Correspondence to: Dr Jiping Su, Department of OtolaryngologyHead and Neck Surgery, First Affiliated Hospital of Guangxi Medical University, 6 Shuang Yong Road, Nanning 530021, P.R. China E-mail:ymsu2@126.com

*Contributed equally

Key words: DNA methylation, secreted protein, acidic and rich in cysteine, head and neck squamous cell carcinoma, tumor suppressor genes

\section{Introduction}

Epidemiological studies show that head and neck squamous cell carcinoma (HNSCC) is the sixth most commonly occurring human neoplasm and includes cancers of the oral cavity, larynx, nasal passages, pharynx and salivary glands. HNSCC accounts for approximately $10 \%$ of the total cancer burden in men in Europe (1). In 2008, there were 47,560 estimated new cases of head and neck cancers in the US, with 11,260 fatalities (2). Although certain independent risk factors for the incidence of HNSCC such as alcohol and tobacco exposure or a family history of malignancy have been revealed $(3,4)$, complete molecular pathogenesis in these human malignancies has yet to be adequately elucidated.

Secreted protein, acidic and rich in cysteine (SPARC)has generated considerable interest as a tumor-associated protein for its diverse actions and complex functions. An enhanced expression of SPARC is associated with a highly aggressive tumor phenotype in melanomas and gliomas, as supported by previous functional verification (5-8). However, other studies reported that SPARC acts as a tumor suppressor gene (TSG) in pancreatic adenocarcinoma, acute myeloid leukemia and ovarian and colorectal carcinomas (9-12). Furthermore, recent studies have shown that SPARC mediates the interaction between cells and the extracellular environment as a matricellular protein and is associated with chemotherapy sensitivity $(13,14)$. However, the role played by SPARC in the tumorigenesis of various common HNSCCs remains to be clarified. The present study aimed to investigate the mechanism underlying the abnormal expression of SPARC in laryngeal and hypopharyngeal carcinomas, the two most common HNSCCs. The clinical significance of aberrant promoter methylation was also investigated.

\section{Materials and methods}

HNSCC cell lines, primary tumors and matched specimens of normal epithelia. Human laryngeal carcinoma cell line Hep-2 was maintained in our laboratory. The cancer cell lines Tu212 and Tu686 were established from primary hypopharyngeal carcinoma, and primary tongue carcinomas, respectively, from the University of Texas, M.D. Anderson 
Cancer Center (Houston, TX, USA), as previously described (15), together with HNSCC cell lines M2E and M4E were kindly provided by Dr He Zhu (XiangYa Central Experiment Laboratory, Changsha, China). A total of 51 primary tumor biopsies of laryngeal and hypopharyngeal carcinoma, with 9 samples of normal epithelia from areas adjacent to the tumors, were included in the present study. The tumor biopsies and matched normal epithelia were obtained at the Department of Otolaryngology-Head and Neck Surgery, First Affiliated Hospital of Guangxi Medical University (Nanning, China), between July 2008 and December 2009. Tissues from the patients were immediately frozen in liquid nitrogen and stored at $-180^{\circ} \mathrm{C}$ until required. Diagnoses were established according to the World Health Organization classification. None of the patients had been administered with preoperative radiation or chemotherapy. Formal written consent was obtained from all patients and approval was obtained from the local ethics committee.

Semi-quantitative reverse transcription polymerase chain reaction $(R T-P C R)$. Total RNA of the cell lines, primary tumor biopsies and adjacent normal epithelia were isolated with TRIzol reagent (Invitrogen, Carlsbad, CA, USA). First strand cDNA was synthesized with M-MLV reverse transcriptase (Promega, Madison, WI, USA) according to the manufacturer's instructions. Total RNA $(2 \mu \mathrm{g})$ was used for each reaction. The primer sequences used were previously reported (9); forward: 5'-AAGCTCACTGGCATGGCCTT-3' and reverse: 5'-CTCTCTTCCTCTTGTGCTCTTG-3' at $61^{\circ} \mathrm{C}$ for $30 \mathrm{sec}$ and $72^{\circ} \mathrm{C}$ for $1 \mathrm{~min}$ at 24 cycles (GAPDH). PCR was carried out in a total volume of $25 \mu 1$. The PCR mixture contained 10 pmol of each primer, 100 pmol of deoxynucleoside triphosphate, 1X PCR buffer, 1 unit of ExTaq HS polymerase (Takara), and $2 \mu 1$ of cDNA. The amplified PCR products were then identified on $2 \%$ agarose gels. Images of ethidium bromide-stained agarose gels were acquired using a CCD camera (Bio-Rad, Hercules, CA, USA) and semi-quantitative analysis was performed using Quantity-One software, version 4.4.0 (Bio-Rad).

Sodium bisulphite modification of genomic DNA. Highmolecular weight genomic DNA was extracted from cell lines and biopsies using a conventional phenol/chloroform method. The sodium bisulphite modification procedure was a slight modification of the protocol previously reported (16). Briefly, $500 \mathrm{ng}$ of genomic DNA was denatured in $0.3 \mathrm{M} \mathrm{NaOH}$ for $15 \mathrm{~min}$ at $37^{\circ} \mathrm{C}$, and then mixed with 2 volumes of $2 \%$ low melting point agarose gels. Agarose/DNA mixtures were then pipetted into chilled mineral oil to form agarose beads. Aliquots of $200 \mu \mathrm{l}$ of $5 \mathrm{M}$ bisulphite solution (2.5 M sodium metabisulphite and $100 \mathrm{mM}$ hydroquinone; Sigma, St. Louis, MO, USA) were added to each tube containing a single bead. The bisulphite reaction was then carried out by incubating the reaction mixture for $4 \mathrm{~h}$ at $50^{\circ} \mathrm{C}$ in the dark. Treatments were stopped by equilibration against $1 \mathrm{ml}$ of TE buffer followed by desulphonation in $500 \mu 1$ of $0.2 \mathrm{M} \mathrm{NaOH}$. Finally, the beads were washed with $1 \mathrm{ml}$ of $\mathrm{H}_{2} \mathrm{O}$ and directly used for PCRs.

Methylation-specific PCR (MSP). The methylation status of the SPARC promoter region was determined by MSP. Primers distinguishing unmethylated (U) and methylated (M) alleles were designed to amplify the sequence within the $\mathrm{CpG}$ island around exon 1 of SPARC, as previously described (9). Each MSP reaction contained $20 \mathrm{ng}$ of sodium bisulphite-modified DNA, 10 pmol of each primer, 100 pmol of deoxynucleoside triphosphate, 1X PCR buffer, and 1 unit of ExTaq HS polymerase in a final reaction volume of $25 \mu$ l. Cycling conditions were as previously described (9). PCR products were separated on $2 \%$ agarose gels, stained with ethidium bromide and visualized under UV illumination. For cases with borderline results, PCR analyses were repeated.

5-Aza-2'-deoxycytidine treatment (5-Aza-dC). The human laryngeal carcinoma cell line Hep-2 and hypopharyngeal carcinoma cell line Tu212 were selected to perform 5-aza-dC treatment. Cells were seeded into 6-well plates with a density of $2 \times 10^{5}$ cells/well. Medium containing 0, 5 or $10 \mu \mathrm{M}$ DNA methyltransferase inhibitor 5-aza-dC (Sigma) was added $24 \mathrm{~h}$ later and replaced every $24 \mathrm{~h}$. After $72 \mathrm{~h}$ of demethylation treatment, the restoration of SPARC expression was examined by RT-PCR, as mentioned above.

Bisulphite genomic sequencing (BGS). Sodium bisulphitemodified DNA was subjected to PCR with primers designed to amplify nucleotides from -29 to +191 bp relative to the transcription start site of the SPARC gene, as previously described (9). PCR was carried out in a total volume of $25 \mu 1$, containing $20 \mathrm{ng}$ of sodium bisulphite-modified DNA as a template, 10 pmol of each primer, 100 pmol of deoxynucleoside triphosphate, 1X PCR buffer, and 1 unit of ExTaq HS polymerase (Takara). PCR products were then gel-purified and cloned using the pMD18-T vector (Takara) and JM109 competent E. coli cells. Colonies were grown on agar plates and five colonies of each sample were randomly selected. Plasmids were then isolated and purified. Sequencing was carried out using a BigDye terminator cycle sequencing kit 3.0 (Applied Biosystems, Carlsbad, CA, USA) on an ABI 3100 sequencer according to the manufacturer's instructions.

Statistical analysis. An independent sample t-test was used to compare transcription expression levels between methylated tumor tissues and normal epithelia, or between methylated and unmethylated tumor tissues. The correlation between numbers of methylated samples and clinicopathological characteristics was analyzed by the $\chi^{2}$ or Fisher's exact test. $\mathrm{P}<0.05$ was considered to be statistically significant.

\section{Results}

Expression and methylation status of SPARC in cancer cell lines. Initially, transcription levels of SPARC were examined by RT-PCR in various HNSCC cell lines, including laryngeal and hypopharyngeal carcinoma cell lines. SPARC expression was undetectable in $100 \%$ (5/5) of HNSCC cell lines. However, SPARC was expressed at an easily detected level in normal epithelial specimens (Fig. 1A). The methylation status of SPARC was investigated by an MSP assay, and aberrant promoter methylation was detected in all of the cell lines, but not in any of the normal epithelial specimens (Fig. 1B). 
A

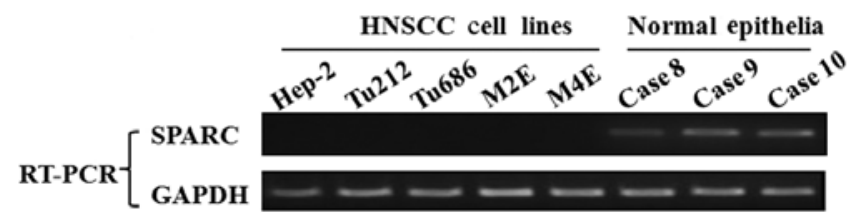

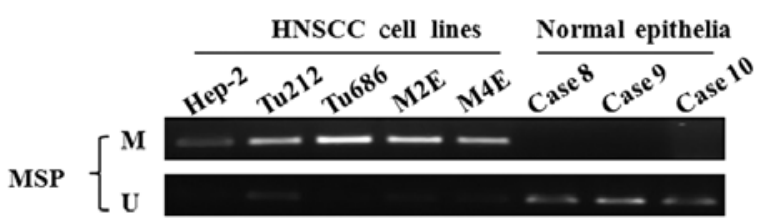

Figure 1. Expression and methylation status of SPARC in HNSCC cell lines. (A) RT-PCR analysis of SPARC transcription expression in HNSCC cell lines. Glyceraldehyde-3-phosphate dehydrogenase (GAPDH) was used as an internal control. (B) The methylation status of the SPARC promoter region was determined using MSP analysis in the HNSCC cell lines. Parallel amplification reactions were performed using primers specific for methylated (M) and unmethylated (U) sequences by MSP.

A

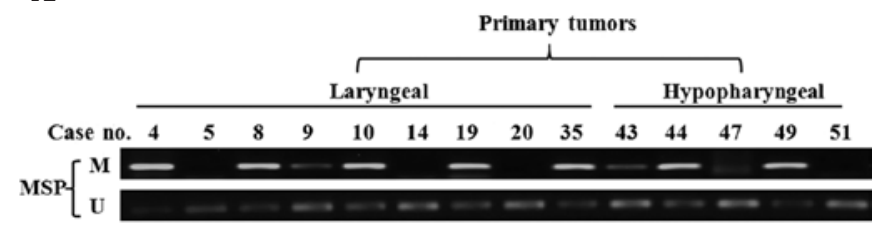

B

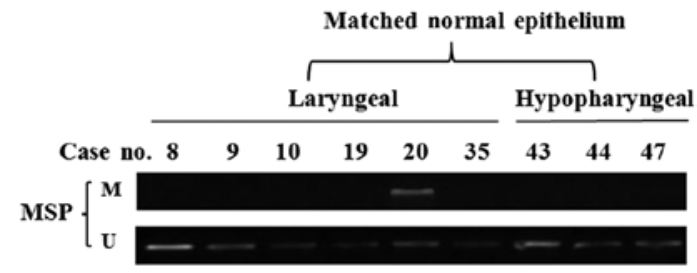

C

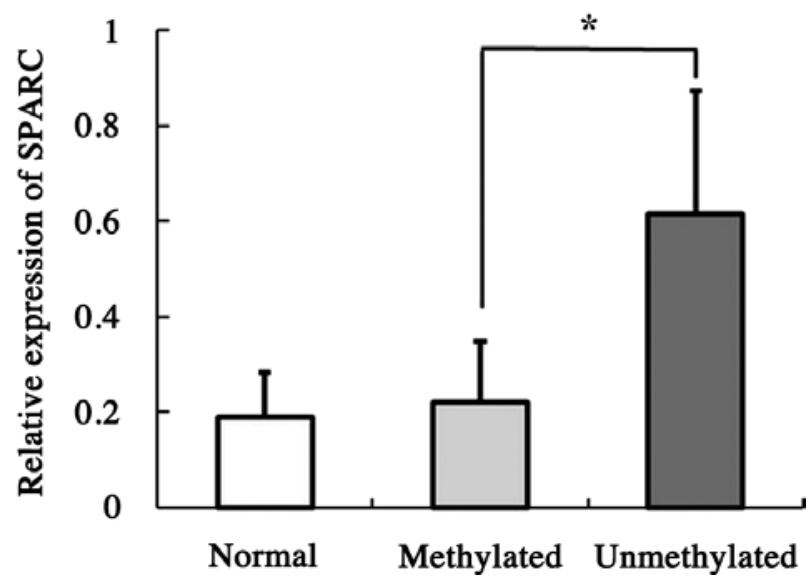

Figure 2. Methylation status of SPARC in primary tumors and corresponding transcription levels; (A) Representative results of MSP analysis in laryngeal and hypopharyngeal carcinoma, including 9 laryngeal and 5 hypopharyngeal carcinoma cases. (B) MSP results of 9 normal epithelial samples are also shown. (C) SPARC expression was quantified by semi-quantitative RT-PCR and standardized with GAPDH expression. The histogram shows the ratios of the intensities of SPARC and GAPDH signals. The data are shown as mean $\pm \mathrm{SD} ;{ }^{*} \mathrm{p}<0.05$.

Transcription levels and aberrant DNA methylation of SPARC in primary tumors of laryngeal and hypopharyngeal carcinoma. The methylation status of the SPARC promoter was detected in 51 primary tumor biopsies. The MSP assay showed that SPARC promoter was hypermethylated in $56.1 \%(23 / 41)$ of laryngeal carcinoma and 70.0\% (7/10) of hypopharyngeal carcinoma specimens (Fig. 2A), but only in $11.1 \%$ (1/9) of samples of normal epithelia (Fig. 2B). In order to evaluate whether methylation is involved in the down-regulation of SPARC in laryngeal and hypopharyngeal carcinoma, transcription levels in methylated tumor, unmethylated tumor and normal epithelial tissues were examined. The transcription expression levels of SPARC in methylated-tumor tissues were found to be significantly lower than those of unmethylated tumor tissues (p<0.05, Fig. 2C), but no significant difference was observed between primary tumors and normal epithelia. This negative result may be attributed either to a smaller number of normal epithelial samples as compared to that required for statistical analysis or to an enhanced stromal expression of SPARC in primary tumors, as previously described (9).

Bisulphite genomic sequence analysis of $C p G$ sites in the SPARC promoter region. Bisulphite genomic sequencing was used to determine the detailed methylation status of the SPARC promoter region -29 to +191 bp relative to the transcription start site of the SPARC gene in 2 carcinoma cell lines,
3 laryngeal carcinoma cases and 1 hypopharyngeal carcinoma case with their matched normal epithelia. The majority of the CpG sites were heavily methylated in cancer cell lines and primary tumors, but almost all of the $\mathrm{CpG}$ sites showed nonmethylation in matched normal epithelia. Fig. 3A shows the typical CpG island spanning the SPARC exon 1 and Fig. 3B shows representative results of the BGS analysis.

Restoration of SPARC expression following pharmacological demethylation treatment. To determine whether SPARC is inactivated by aberrant methylation, laryngeal and hypopharyngeal carcinoma cell lines Hep-2 and Tu212 were treated with the methyltransferase inhibitor 5-aza-dC and then examined using RT-PCR analysis. SPARC expression was restored by pharmacological demethylation treatment in the Hep- 2 cell line by 5 or $10 \mu \mathrm{M}$ 5-aza-dC, but not in the Tu212 cell line (Fig. 4).

Correlation between methylation status and clinicopathological characteristics in laryngeal and hypopharyngeal carcinoma. An analysis of the association of SPARC promoter hypermethylation and clinicopathological characteristics of laryngeal and hypopharyngeal carcinoma patients revealed no significant correlation between aberrant methylation and age, location or T stage. However, SPARC promoter hypermethylation was found to be associated with lymph node metastasis $(\mathrm{p}<0.01)$ (Table I). 
A

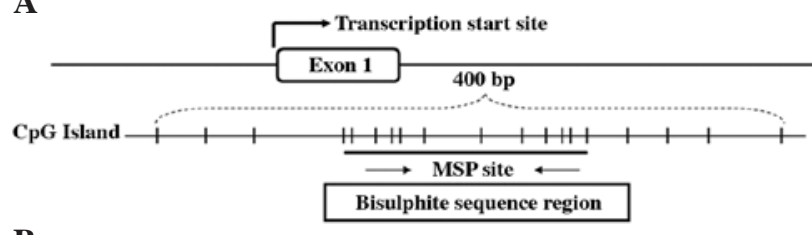

B

Hep-2

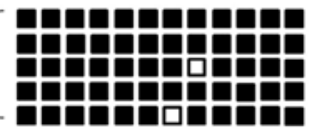

Case 8
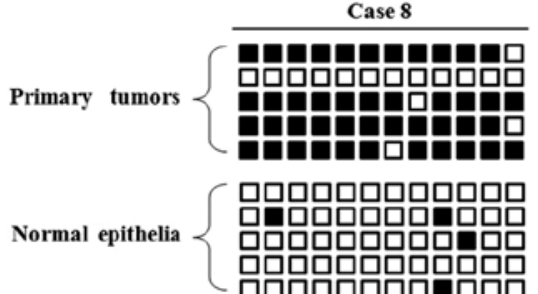

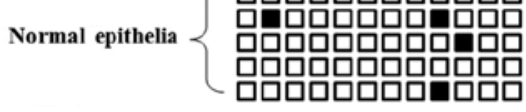

Figure 3. Bisulphite genomic sequencing. (A) Locations of the $12 \mathrm{CpG}$ sites analyzed and primers used in the SPARC promoter region. Vertical lines indicate individual cytosine residues of the $\mathrm{CpG}$ sites. Methylation-specific PCR and bisulphite genomic sequencing (BGS) regions are shown. (B) BGS of the methylation status of SPARC in 2 cancer cell lines, 2 laryngeal carcinoma cases and their matched normal epithelia. Five randomly selected clones were sequenced for each sample. Open squares are unmethylated cytosines, and closed squares are methylated cytosines.
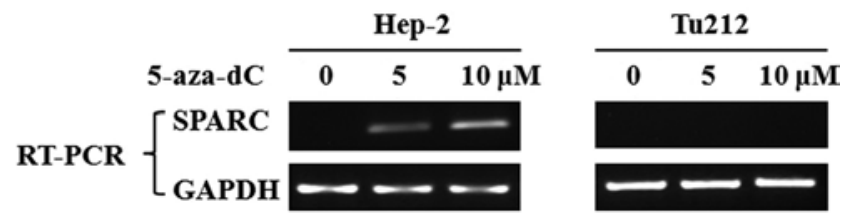

Figure 4. Restoration of SPARC following pharmacological demethylation treatment. After $72 \mathrm{~h}$ of 5 -aza-dC pretreatment, SPARC expression was examined using RT-PCR analysis. GAPDH was used as a control for RNA quality and loading.

Table I. Correlation between clinicopathological characteristics and SPARC promoter methylation.

\begin{tabular}{lccc}
\hline & No. & Promoter methylation & p-value \\
\hline Age & & & \\
$<60$ & 29 & $51.7 \%(15 / 29)$ & 0.237 \\
$\geq 60$ & 22 & $68.2 \%(15 / 22)$ & \\
Location & & & \\
$\quad$ Larynx & 41 & $56.1 \%(23 / 41)$ & 0.423 \\
$\quad$ Hypopharynx & 10 & $70 \%(7 / 10)$ & \\
T stage & & & \\
T1-2 & 16 & $50.0 \%(8 / 16)$ & 0.387 \\
T3-4 & 35 & $62.9 \%(22 / 35)$ & \\
N stage & & & \\
N0 & 21 & $28.6 \%(6 / 21)$ & $\mathbf{0 . 0 0 0}$ \\
N1-2 & 30 & $80.0 \%(24 / 30)$ & \\
\hline
\end{tabular}

Statistically significant values are in bold type; ${ }^{\mathrm{a}} \mathrm{p}$-values are from the $\chi^{2}$ or Fisher's exact test.

\section{Discussion}

In addition to genetic changes, the transcriptional inactivation of TSGs in human tumors by promoter hypermethylation has been extensively studied. Methylation-associated silencing of TSGs in HNSCC has been reported (17-19). However, further identification of cancer-related genes is required in order to gain a better understanding of the precise molecular mechanism underlying the development of HNSCC.

As a prototypic matricellular protein, SPARC plays a significant role in carcinogenesis, tumor progression, invasion and metastasis by affecting the cell shape, differentiation, attachment, migration, proliferation and growth factor activity. The silencing of SPARC in various HNSCC cell lines, including those of laryngeal and hypopharyngeal carcinoma, was first observed in the present study (Fig. 1A). The MSP assay also indicated that the CpG island of the SPARC promoter was frequently methylated in these cell lines (Fig. 1B). The methylation status of the SPARC promoter in primary tumor biopsies of laryngeal and hypopharyngeal carcinoma was investigated. High frequencies of SPARC promoter hypermethylation $(58.8 \%, 30 / 51)$ were detected in primary tumors, but were almost entirely absent from adjacent normal epithelia. Notably, aberrant methylation was found even in early stage (T1 or T2) cases $(50.0 \%, 8 / 16)$. This finding may reflect the fact that hypermethylation of SPARC is a frequent and tumor-specific early event in laryngeal and hypopharyngeal carcinoma. Patients lacking SPARC expression were associated with a poorer prognosis in a survival analysis of colorectal carcinoma (12). In our study, statistical analysis showed that SPARC promoter hypermethylation was associated with lymph node metastasis $(\mathrm{p}<0.01)$. This result suggests that SPARC methylation serves as a biomolecular marker for the diagnosis and prognosis of laryngeal and hypopharyngeal carcinoma.

As with the MSP assay, a subsequent BGS analysis showed that the SPARC promoter was heavily methylated in SPARC-silenced cancer cell lines (Fig. 3B). A heavy degree of methylation was observed in primary tumor biopsies (Fig. 3B), despite the inevitable normal tissue contamination in the biopsy samples without micro-dissection. Furthermore, we showed that SPARC expression was rescued by 5 -aza-dC treatment (Fig. 4). Although demethylation treatment failed to restore SPARC expression in the Tu212 cell line, our results indicated that the epigenetic inactivation of SPARC by aberrant promoter methylation is a significant, if not the only, mechanism contributing to the loss of SPARC expression in laryngeal and hypopharyngeal carcinoma.

The question of whether SPARC is a TSG or an oncogene involved in human cancer remains unresolved, and the precise role played by SPARC in human cancer has yet to be clarified. An enhanced SPARC expression has been observed in oral squamous cell carcinoma and other HNSCC $(20,21)$. On the other hand, the inactivation of SPARC by aberrant DNA methylation has also been reported in pancreatic, ovarian, colon and lung cancers $(9,11,12,22)$. With regards to SPARC function, exogenous SPARC inhibits the proliferation, movement and migration of human cancer cell lines $(11,23)$, which is consistent with the results of an in vivo study (14). A recent study also provided evidence that exogenous SPARC 
enhances the apoptosis of colon cancer cells via the caspase 8 pathway (24).

In conclusion, SPARC is inactivated in laryngeal and hypopharyngeal carcinoma by promoter hypermethylation, which promotes the expression of these HNSCCs. Studies investigating the in-depth mechanism of SPARC are required to determine whether its promoter hypermethylation is likely to serve as a biomolecular indicator for prognosis, as well as whether the selective rescue of SPARC is a promising strategy for increasing the efficacy of cancer therapy in laryngeal and hypopharyngeal carcinoma patients.

\section{Acknowledgements}

This study was supported by the Natural Science Foundation of China (No. 30960416 to Z.Z.) and the Science and Technology Department of Guangxi Province, China (No. 0728052 to Z.Z). We are grateful to Dr Y. Chen (Department of Epidemiology and Health Statistics, Public Health of Guangxi Medical University) for her enthusiastic assistance in the statistical analysis. Dr H. Zhu's (XiangYa Central Experiment Laboratory, Changsha, China) provision of HNSCC cell lines is also acknowledged.

\section{References}

1. Ferlay J, Autier P, Boniol M, Heanue M, Colombet M and Boyle P: Estimates of the cancer incidence and mortality in Europe in 2006. Ann Oncol 18: 581-592, 2007.

2. Jemal A, Siegel R, Ward E, Hao Y, Xu J, Murray T and Thun MJ: Cancer statistics,. CA Cancer J Clin 58: 71-96, 2008.

3. DeRienzo DP, Greenberg SD and Fraire AE: Carcinoma of the larynx. Changing incidence in women. Arch Otolaryngol Head Neck Surg 117: 681-684, 1991.

4. Chen YJ, Chang JT, Liao CT, Wang HM, Yen TC, Chiu CC, $\mathrm{Lu}$ YC, Li HF and Cheng AJ: Head and neck cancer in the betel quid chewing area: recent advances in molecular carcinogenesis Cancer Sci 99: 1507-1514, 2008.

5. Rempel SA, Golembieski WA, Ge S, Lemke N, Elisevich K, Mikkelsen T and Gutierrez JA: SPARC: a signal of astrocytic neoplastic transformation and reactive response in human primary and xenograft gliomas. J Neuropathol Exp Neurol 57: 1112-1121, 1998.

6. Seno T, Harada H, Kohno S, Teraoka M, Inoue A and Ohnishi T: Downregulation of SPARC expression inhibits cell migration and invasion in malignant gliomas. Int J Oncol 34: 707-715, 2009.

7. Ledda F, Bravo AI, Adris S, Bover L, Mordoh J and Podhajcer OL: The expression of the secreted protein acidic and rich in cysteine (SPARC) is associated with the neoplastic progression of human melanoma. J Invest Dermatol 108: 210-214, 1997.

8. Horie K, Tsuchihara M and Nakatsura T: Silencing of secreted protein acidic and rich in cysteine inhibits the growth of human melanoma cells with G arrest induction. Cancer Sci 101: 913-919, 2010.

9. Sato N, Fukushima N, Maehara N, Matsubayashi H, Koopmann J, $\mathrm{Su} \mathrm{GH}$, Hruban RH and Goggins M: SPARC/osteonectin is a frequent target for aberrant methylation in pancreatic adenocarcinoma and a mediator of tumor-stromal interactions. Oncogene 22: 5021-5030, 2003.
10. Di Martino JF, Lacayo NJ, Varadi M, Li L, Saraiya C, Ravindranath Y, Yu R, Sikic BI, Raimondi SC and Dahl GV: Low or absent SPARC expression in acute myeloid leukemia with MLL rearrangements is associated with sensitivity to growth inhibition by exogenous SPARC protein. Leukemia 20: 426-432, 2006.

11. Socha MJ, Said N, Dai Y, Kwong J, Ramalingam P, Trieu V, Desai N, Mok SC and Motamed K: Aberrant promoter methylation of SPARC in ovarian cancer. Neoplasia 11: 126-13512, 2009.

12. Yang E, Kang HJ, Koh KH, Rhee H, Kim NK and Kim H: Frequent inactivation of SPARC by promoter hypermethylation in colon cancers. Int J Cancer 121: 567-575, 2007.

13. Cheetham S, Tang MJ, Mesak F, Kennecke H, Owen D and Tai IT: SPARC promoter hypermethylation in colorectal cancers can be reversed by 5-Aza-2'deoxycytidine to increase SPARC expression and improve therapy response. $\mathrm{Br} \mathrm{J}$ Cancer 98: 1810-1819, 2008.

14. Bull-Phelps SL, Carbon J, Miller A, Castro-Rivera E, Arnold S, Brekken RA and Lea JS: Secreted protein acidic and rich in cysteine as a regulator of murine ovarian cancer growth and chemosensitivity. Am J Obstet Gynecol 200: 180-187, 2009.

15. Zhang X, Chen ZG, Choe MS, Lin Y, Sun SY, Wieand HS, Shin HJ, Chen A, Khuri FR and Shin DM: Tumor growth inhibition by simultaneously blocking epidermal growth factor receptor and cyclooxygenase- 2 in a xenograft model. Clin Cancer Res 11: 6261-6269, 2005.

16. Olek A, Oswald J and Walter J: A modified and improved method for bisulphite based cytosine methylation analysis. Nucleic Acids Res 24: 5064-5066, 1996

17. Maruya S, Issa JP, Weber RS, Rosenthal DI, Haviland JC, Lotan R and El-Naggar AK: Differential methylation status of tumor-associated genes in head and neck squamous carcinoma: incidence and potential implications. Clin Cancer Res 10: 3825-3830, 2004

18. Bennett KL, Karpenko M, Lin MT, Claus R, Arab K, Dyckhoff G, Plinkert P, Herpel E, Smiraglia D and Plass C: Frequently methylated tumor suppressor genes in head and neck squamous cell carcinoma. Cancer Res 68: 4494-4499, 2008.

19. Bennett KL, Lee W, Lamarre E, Zhang X, Seth R, Scharpf J, Hunt $J$ and Eng C: HPV status-independent association of alcohol and tobacco exposure or prior radiation therapy with promoter methylation of FUSSEL18, EBF3, IRX1, and SEPT9, but not SLC5A8, in head and neck squamous cell carcinomas. Genes Chromosomes Cancer 49: 319-326, 2010.

20. Choi P, Jordan CD, Mendez E, Houck J, Yueh B, Farwell DG, Futran $\mathrm{N}$ and Chen $\mathrm{C}$ : Examination of oral cancer biomarkers by tissue microarray analysis. Arch Otolaryngol Head Neck Surg 134: 539-546, 2008.

21. Chin D, Boyle GM, Williams RM, Ferguson K, Pandeya N, Pedley J, Campbell CM, Theile DR, Parsons PG and Coman WB: Novel markers for poor prognosis in head and neck cancer. Int J Cancer 113: 789-797, 2005.

22. Suzuki M, Hao C, Takahashi T, Shigematsu H, Shivapurkar N, Sathyanarayana UG, Iizasa T, Fujisawa T, Hiroshima K and Gazdar AF: Aberrant methylation of SPARC in human lung cancers. Br J Cancer 92: 942-948, 2005.

23. Chen G, Tian X, Liu Z, Zhou S, Schmidt B, Henne-Bruns D, Bachem $M$ and Kornmann $M$ : Inhibition of endogenous SPARC enhances pancreatic cancer cell growth: modulation by FGFR1-III isoform expression. Br J Cancer 102: 188-195, 2010.

24. Tang MJ and Tai IT: A novel interaction between procaspase 8 and SPARC enhances apoptosis and potentiates chemotherapy sensitivity in colorectal cancers. J Biol Chem 282: 34457-34467, 2007. 